\title{
Multiple-Beam Laser Guidance-Based Microscope for Patterning Adult Cardiomyocytes
}

\author{
Lucas Schmidt ${ }^{1}$, Zhonghai Wang ${ }^{1}$, Nick Erdman ${ }^{1}$, Tong Ye ${ }^{1}$, Thomas K. Borg ${ }^{1,2}$, and Bruce Z. Gao ${ }^{2}$ \\ 1. Department of Bioengineering, Clemson University, Clemson, SC, USA \\ 2. Department of Regenerative Medicine and Cell Biology, Medical University of South Carolina, \\ Charleston, SC, USA
}

Traditional cell-culture techniques lack the spatial control of single cells that is necessary to recreate the cell-cell contact arrangement found in tissue. Since Ashkins developed optical force-based cellmanipulation techniques [1], the laser microbeam has been used in a microscopic system to explore various biological interactions at molecular and cellular levels. These explorations of a single cell or subcellular organelle involve trapping (using a laser-tweezers microscope [2]) and guidance (using a laser-guided direct writing microscope [3, 4]). When laser tweezers are used, a high numerical aperture (NA) microscope objective generates a strongly focused laser beam, which 3D traps a particle, such as a biological cell, in the beam's focal point. In laser-guided direct writing, a low NA microscope objective generates a weakly focused laser beam, which traps a particle in the beam axis and guides it to move along the direction of beam propagation.

Based on these techniques, we have developed a laser cell-micropatterning system in which the laser beam is focused in a transition state between generating an optical trap and optical guidance [5]. Using this system, a single biological cell can be trapped in a typical (e.g., 30mm) cell culture dish and patterned onto a designated cell culture niche with very high spatial and temporal resolution [6-8]. However, this and the other currently available laser-based single-cell-manipulation techniques can be used to pattern only spherical cells, not irregularly shaped cells such as rod-shape cardiomyocytes. The work stems from the use of a spatial light modulator (SLM) loaded with a computer generated phase map to shape a single laser beam into multiple microbeams analogous to techniques used in holographic optical tweezers (HOT) [9]. In contrast to the optical configuration in HOT, our system uses a low NA objective to produce multiple weakly focused laser microbeams in our multiple beam laser guidance system. Based on the cell image acquired during laser cell patterning, the multiple beams can be distributed around the outer contour of an irregularly shaped cell to achieve accurate cell patterning. In addition to describing the principle and practice of the system design, here we present what is to our knowledge the first achievement of patterning large, irregularly (rod) shaped adult rat cardiomyocytes (ACMs) in an end-to-end connected alignment to replicate the in vivo heart-muscle structure without use of substrate surface modifications that may interfere with in vivo-like cell-cell and cell-extracellular matrix interactions.

The basic structure of the multiple-beam laser cell-patterning system is schematically shown in Figure 1. The cell suspension was loaded into the microfluidics-based cell feeding system built in the celldeposition chamber, with a microfluidic channel width of $200 \mu \mathrm{m}$. The guidance region (also the imaging region of the objective) was initially focused onto the outlet of the cell-feeding microfluidic channel by controlling the movement of the cell-deposition chamber. A target cell was manually selected and continuously traced by the imaging system with a lab-developed cell-recognition algorithm to configure the multiple beams (Figure 2) in real time through an SLM according to the shape of the 
selected cell. Once a cell is trapped by the multiple beams, it was instantly propelled downward along the optical axis and thus gradually pushed out of focus. It was then be moved towards the imaging region of the objective by moving the chamber up during guidance for continuous image tracking, which generated a relative motion of the cell towards the bottom. Simultaneously, the cell was moved to target the substrate horizontally by moving the motorized stage. This move was controlled quasiautomatically through an Xbox360 controller with customized software. The feedback obtained from the cell image in real time was used to modify the multiple-beam configuration to maintain optimal guidance. The final cell deposition process with find spatial-orientation adjustment (Figure 3) was achieved manually.

\section{References:}

[1] Ashkin, A., Ieee Journal of Selected Topics in Quantum Electronics 6 (2000), p. 841-856.

[2] Svoboda, K. and S.M. Block, Annual Review of Biophysics and Biomolecular Structure 23 (1994), p. 247-285.

[3] Odde, D.J. and M.J. Renn, Trends in Biotechnology 17 (1999), p. 385-389.

[4] Odde, D.J. and M.J. Renn, Biotechnology and Bioengineering 67 (2000), p. 312-318.

[5] Ma, Z., et al., Light: Science \& Applications 2 (2013), p. e68.

[6] Erdman, N., et al., Biofabrication 6 (2014).

[7] Ma, Z., et al., Biofabrication 3 (2011), p. 0341071-8.

[8] Pirlo, R.K., et al., Review of Scientific Instruments 82 (2011).

[9] Padgett, M. and R. Di Leonardo, Lab on a Chip 11 (2011), p. 1196-1205.

[9] The authors acknowledge funding from the National Institutes of Health, Grant Number P20GM103444 and R01HL124782; and from American Heart Association, Grant Number 14GRNT20520004.

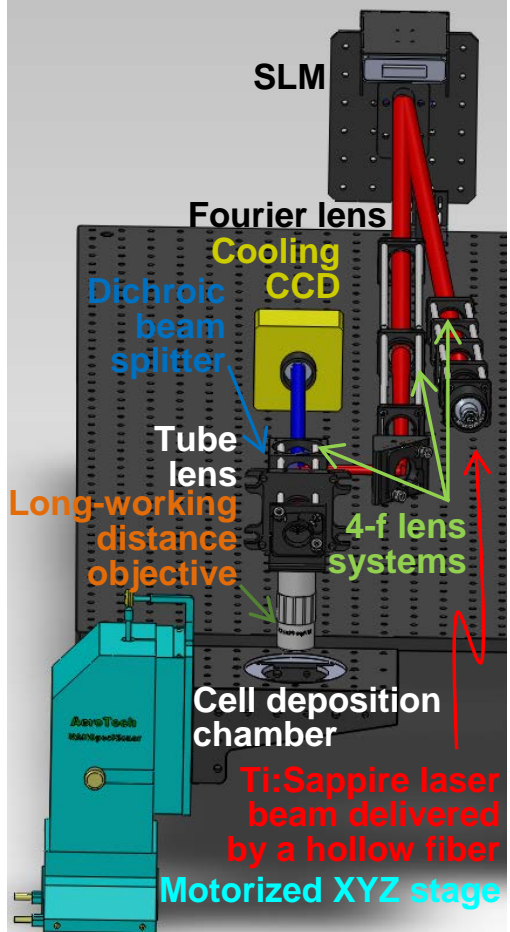

Figure 1. Schematics of the SLM-based multiple-beam laser guidance system

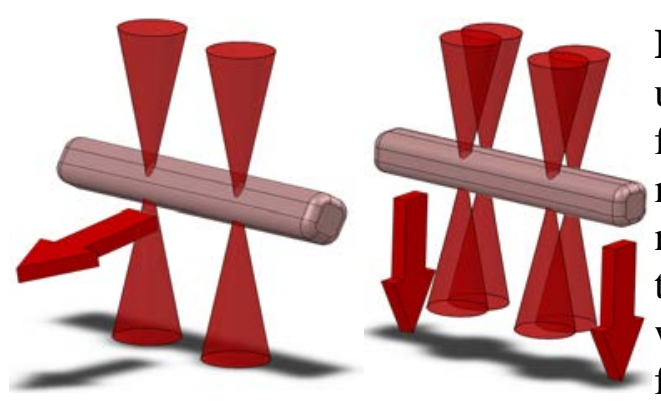

Figure 2. A two-beam setup was used to guide an adult myocyte from the outlet of the cell feeding microfluidic channel to a position near the cell deposition site, and then a four-beam configuration was used to deposit the cell with fine spatial adjustment.

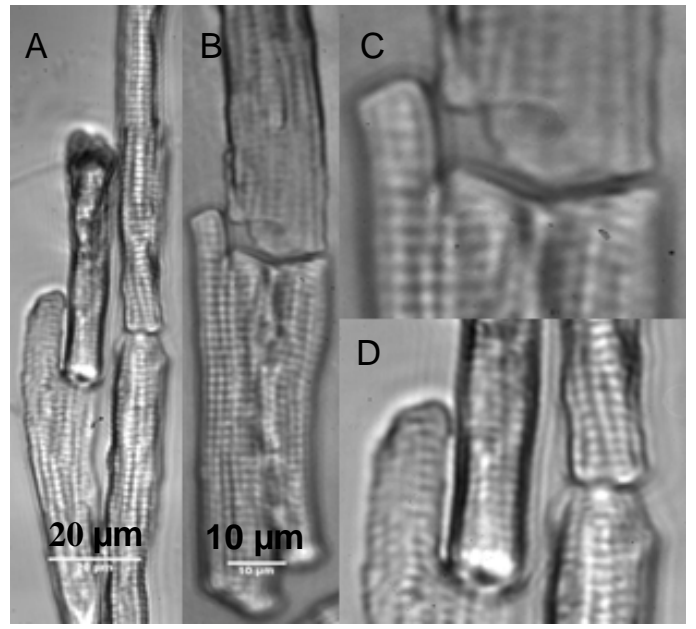

Figure 3. Typical images of end-to-end connected adult cardiomyocytes captured from the laser guidance-based cell patterning microscope. $\mathrm{C}$ and $\mathrm{D}$ are zoomed in images of $\mathrm{B}$ and $\mathrm{A}$, showing the fine alignment at the cell interface between two cells with irregularly shaped cell ends. 